\title{
BMJ Open Clinical outcomes and associated predictors of early intervention in autism spectrum disorder: a study protocol
}

\author{
Anne Masi (D , ${ }^{1}$ Cheryl Dissanayake, ${ }^{2}$ Tasha Alach, ${ }^{3}$ Kate Cameron, ${ }^{4}$ \\ Kathryn Fordyce, ${ }^{5}$ Grace Frost, ${ }^{6}$ Rachel Grove, ${ }^{7}$ Helen Heussler, ${ }^{8}$ Natalie Silove, ${ }^{9}$ \\ Rhylee Sulek, ${ }^{10}$ Madonna Tucker, ${ }^{11,12}$ Katrina Williams, ${ }^{13,14}$ \\ Valsamma Eapen (id) 1,15,16
}

To cite: Masi A, Dissanayake C, Alach T, et al. Clinical outcomes and associated predictors of early intervention in autism spectrum disorder: a study protocol. BMJ Open 2021;11:e047290. doi:10.1136/ bmjopen-2020-047290

- Prepublication history for this paper is available online To view these files, please visit the journal online (http://dx.doi org/10.1136/bmjopen-2020047290).

Received 26 November 2020 Accepted 20 July 2021

Check for updates

(c) Author(s) (or their employer(s)) 2021. Re-use permitted under CC BY-NC. No commercial re-use. See rights and permissions. Published by BMJ.

For numbered affiliations see end of article.

Correspondence to Professor Valsamma Eapen; v.eapen@unsw.edu.au

\section{ABSTRACT}

Introduction Research highlights the importance of early intervention for children with autism spectrum disorder with better outcomes associated with earlier access to early intensive intervention (EII) programmes. However, there is significant variability in response to Ell despite children receiving the same programmes.

Methods and analysis A prospective, multisite cohort study using a pre-post design assesses the predictors of early intervention outcomes for children who receive Ell through six early intervention services (Autism Specific Early Learning and Care Centres, ASELCCs) across Australia. Child and family characteristics at entry to and exit from ASELCCs are ascertained using measures of autism symptoms (Autism Diagnostic Observation Schedule-2; Social Communication Questionnaire); cognitive, language and developmental skills (Mullen Scale of Early Learning); adaptive function (Vineland Adaptive Behaviour Scale-second Edition); behaviours (Child Behaviour Checklist-1.5 to 5 years; Restricted Repetitive Behaviour Scale); parental stress (Parent Stress Index-4 Short Form); quality of life (Quality of Life in Autism Scale) and a semistructured family history questionnaire for sociodemographic, family and psychosocial characteristics. Characteristics at entry are used as predictors of outcome at exit following Ell approximately 12 months later. The change in score from baseline to exit will be the primary outcome of interest. The mediating role of family and psychosocial factors will also be considered. Ethics approval University of New South Wales Human Research Ethics Committee (HC14267).

Dissemination of results Findings will be published in peer-reviewed journals and presented at conferences. A report summarising data and the interpretation of data will be published.

\section{INTRODUCTION}

Autism spectrum disorder (ASD) is characterised by difficulties in social communication and restricted, repetitive and stereotyped patterns of behaviours and interests. ${ }^{1}$ Overall prevalence estimates vary widely from $0.8 \%$ of
Strengths and limitations of this study

- Multisite study with a large sample size on early intervention in autism.

- Unique dataset with detailed individual child profile ascertained at baseline to predict response to early intervention in autism spectrum.

- A cohort of preschool children on autism spectrum with comprehensive assessment of individual clinical and behavioural characteristics as well as family stress and quality of life.

- This is a pre-post study and there is no control group.

- The nature and duration of intervention are variable across the cohort

preschool-aged children, to $1 \%$ of the general child population and as high as 1 in 54 in a population of the 8-year-old children. ${ }^{2-4}$ ASD is a heterogeneous condition with significant variability in clinical presentation, functional impact and response to intervention. ${ }^{5}$ With numerous neurodevelopmental genes and non-genetic factors implicated in the aetiology, ASD is recognised to be 'autisms' or a spectrum of conditions that affect individuals differently with myriad underlying biological processes and developmental pathways. ${ }^{6}$ Co-occurring conditions, such as intellectual disability and anxiety and medical conditions, also add to the complexity of presentations of ASD. ${ }^{7}$

Previous research highlights the importance of early intervention for ASD-or children on the autism spectrum-with better outcomes associated with earlier access to early intervention programmes ${ }^{8}$ as these allow for the harnessing of the neural connections forming during the early years of life. ${ }^{9}$ However, there is significant variability in 
response to early intervention despite children receiving the same intervention programmes further highlighting the heterogeneity. ${ }^{10}$ While some clinical and behavioural factors have been associated with positive intervention outcomes, ${ }^{11}$ the fact that behaviourally similar children can respond very differently to the same intervention suggests that there are other factors that may have greater predictive value in terms of intervention outcomes. ${ }^{12}$ That is, there may be specific clinical characteristics in addition to family and psychosocial factors, and intervention variables that may determine differential response to intervention and outcomes. ${ }^{12} 13$

This project uses a pre-post design to determine the developmental and behavioural characteristics, and family and psychosocial factors, that are associated with differential intervention outcomes in children on the autism spectrum. In addition to ascertaining whether participation in an autism specific early intervention programme improves overall functioning of children on the autism spectrum, a key aim is to understand the specific determinants of such outcomes. This could then lead to identifying homogeneous subgroups that have specific responses to intervention, as well as developmental trajectories and outcomes. Exploring the associated clinical factors that underpin homogeneous subgroups within ASD is expected to provide valuable insight into the unique child characteristics that determine course and outcome. The findings of this study are also expected to provide much needed information on the matching of clinical characteristics and subgroups with the most effective intervention strategy.

The Cooperative Research Centre for Living with Autism (Autism CRC; https://www.autismcrc.com.au/) was established in 2013 as the world's first national, cooperative research effort focused on autism across the lifespan. The Australian Autism Subtyping Project was initiated by the Autism CRC to evaluate the determinants of outcomes of early intensive intervention (EII) on autism symptoms, cognitive and language level, adaptive functioning and behavioural outcomes of the child and parental quality of life; thereby providing critical evidence base on predictors of intervention outcomes in ASD which has the potential to identify homogeneous subgroups. It is expected that the data resource created from the Autism Subtyping Project will be of interest to both national and international researchers as it represents the largest cohort of children on the spectrum receiving EII in controlled settings. The Autism Subtyping Project is being carried out in conjunction with the Child and Family Outcome Study (CFOS) set up in 2010 and funded by Department of Social Services (DSS), Commonwealth Government of Australia.

\section{Study aims}

The primary aim in this project is to identify, in a cohort of children on the autism spectrum aged 1-6 years, the clinical characteristics at entry to Autism Specific Early Learning and Care Centres (ASELCCs) that predict autism symptoms, cognitive and language ability, adaptive function and behavioural outcomes, as well as the parental quality of life outcomes following 1 year of EII.

The secondary aim is to evaluate the family and psychosocial variables that mediate identified associations between baseline characteristics and child outcomes.

\section{METHODS AND ANALYSIS}

\section{Participant and public involvement}

The study protocol was reviewed for input by autistic adults with an interest in autism research. Future studies involving data generated from this study will be interpreted and disseminated by a consumer research advisory group. Further, stakeholders, including the ASELCCs, families of children on the spectrum, autistic adults and research institutions, will work together to translate the research into positive benefits for the autistic community including raising awareness and capacity building activities.

\section{Study design}

This is a confirmatory, prospective, multicentre, prepost study with one treatment arm and two measurement time points. All children complete a battery of cognitive and ASD behaviour assessments on entry to the ASELCC. Parents or caregivers complete additional questionnaires on child behaviour, parental stress and quality of life, and a questionnaire containing sociodemographic information. Teachers (ASELCC staff) also complete a questionnaire on child behaviour. All assessments and questionnaires are completed again at exit from the centre, approximately 12 months after entry, or at follow-up should the child be continuing in the intervention programme for longer than 12 months.

\section{Study timetable}

The ASELCCs providing EII in a long-day care setting within which the CFOS research and the Autism Subtyping Project are conducted, were established in 2009 with funding from the DSS in each of the six Australian states: New South Wales, Queensland, South Australia, Tasmania, Victoria and Western Australia. Children showing symptoms consistent with ASD who have not yet received a diagnosis are also eligible for enrolment. In addition, each centre provides support to the children's families, undertakes research, and builds capacity within the sector more broadly. This investment was part of the Government's commitment to the National Early Childhood Development Strategy. ${ }^{14}$ Each of the six centres, and their associated research institutions, agreed to contribute data to the Autism CRC's Autism Subtyping Project in 2014 and the study is being conducted from January 2015 to September 2021.

\section{Study sites}

The six ASELCCs represent the study sites (in alphabetical order based on state location): South Western 
Sydney, NSW: KU Marcia Burgess ASELCC (KU Children's Services); Queensland: Queensland ASELCC; Adelaide, SA: Anglicare-SA, Daphne Street Child Care and Specialist Early Learning Centre (Anglicare South Australia Inc); North West Tasmania: North West Tasmania ASELCC (St Giles Society); Victoria: Margot Prior ASELCC, La Trobe University Community Children's Centre (La Trobe University); Perth, WA: First Steps Autism Day Care Midland (The Autism Association of Western Australia).

Each study site has a multidisciplinary team of educators and allied health therapists, including speech pathologists and occupational therapists, and a research affiliate to assist with administration of assessments, data collection, data entry and database management (table 1).

\section{Participants}

To be eligible for an early intervention placement and to attend one of the ASELCCs, a child must be less than 6 years of age, with a diagnosis of ASD or probable ASD like symptoms. Priority is given to children who are eligible to start school in the following year. No exclusion criteria regarding cognitive ability, language level or child behaviour are applied.

\section{Sample size}

The sample size estimation has taken into account the capacity of five ASELCCs to enrol approximately 20 children per year in the early intervention programme. One of the ASELCCs can accommodate up to 44 children. Over a 6-year period, this will yield a sample size of approximately 750 children. This is sample size will allow $90 \%$ power, at a two-sided $5 \%$ significance level, to detect a 0.25 standardised difference in mean intentions scores between intervention and control group at follow-up. This sample size calculation accounts for the clustering effect of sites $(\mathrm{ICC}=0.03)$, assumes $\mathrm{r}=0.70$ between individual measurements between entry and exit, a cluster autocorrelation of 0.70 , and allows for $20 \%$ lost to follow-up.

\section{Procedure}

When the child first enrols at the ASELCC, the centre manager, or a member of the multidisciplinary team of therapists at the ASELCC, will have a discussion with one or both of the child's parents about the research programme and provide the parents with the study participant information sheet. If the family is interested in participating in the research, they will be asked by either the professional staff member or research assistant to sign the consent form. Potential participants are informed that participation is voluntary and a decision not to participate will in no way impact on their attendance at the centre. There are no study advertisements or flyers and no pre-screening measures used.
Each ASELCC conducts an EII programme, with a theoretical background framework based on either one type of EII, such as the Early Start Denver Model or a combination of various programmes or models (table 1). There is significant overlap between the components of each of the six ASELCCs EII programmes as described in table 1. The ASELCC staff comprise a multidisciplinary team of early childhood educators and facilitators, allied health professionals and psychologists. The staff to child ratio at each ASELCC is typically between 1:3 and 1:4. The model of intervention implemented at each site is manualised.

Once families consent to participation in the research programme, they will be provided with a package containing the questionnaires to be completed by one or both parents depending on the questionnaire, and an agreed time will be arranged for the completion of the researcher administered assessments. Data collection is undertaken by either the professional staff of each ASELCC or a research assistant, who have been trained by experts in the field to become familiar with various sets of data collection forms and as well as interview techniques where applicable. Relevant staff also attended formal training to administer the Autism Diagnostic Observation Schedule -Second Edition (ADOS-2). ${ }^{15} 16$ All outcome measures and questionnaires are either administered or collected at the initial assessment (baseline) and at exit from the centre, or at follow-up if the child is attending the centre for longer than 12 months .

Baseline characteristics at entry to ASELCCs included the following measures which will be used as predictors of outcome at exit from the centre following EII approximately 12 months later. Change in score between baseline and approximately 1-year postintervention will be the primary outcome of interest. Consideration will also be given to whether family, psychosocial, and quality of life determinants mediate child outcomes.

All autism-related measures were selected based on their previous psychometric evaluations and use across cultures. Australian national census items were adopted to assess family and psychosocial variables such as place of usual residence, age, sex, level of education, income, country of birth and language spoken at home.

\section{Measures}

Autism symptoms

The ADOS- $2^{15}$ is a semistructured, standardised diagnostic observational assessment used to confirm the Diagnostic and Statistical Manual of Mental Disorders, 5th Edition (DSM-5) ${ }^{1}$ diagnosis and determine the severity of ASD at baseline. To assess further for symptoms of autism, a screening measure is also administered to parents or caregivers. The Social Communication Questionnaire $^{17}$ is a 40 -item (yes/no) parent-report screening measure that evaluates communication, reciprocal social interaction, and restricted and repetitive behaviours and interests which are core diagnostic criteria for ASD. ${ }^{1}$ This questionnaire is included in the 


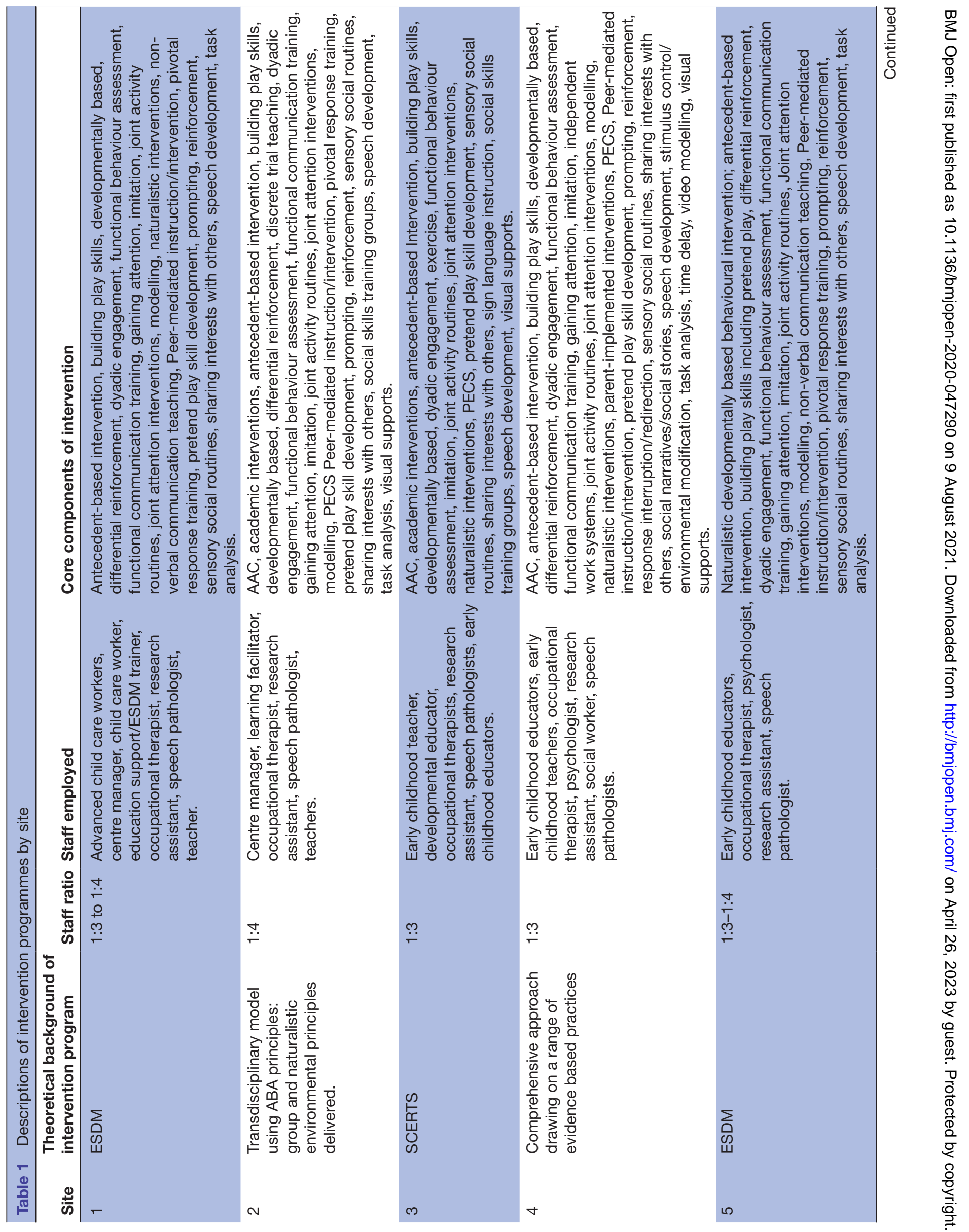




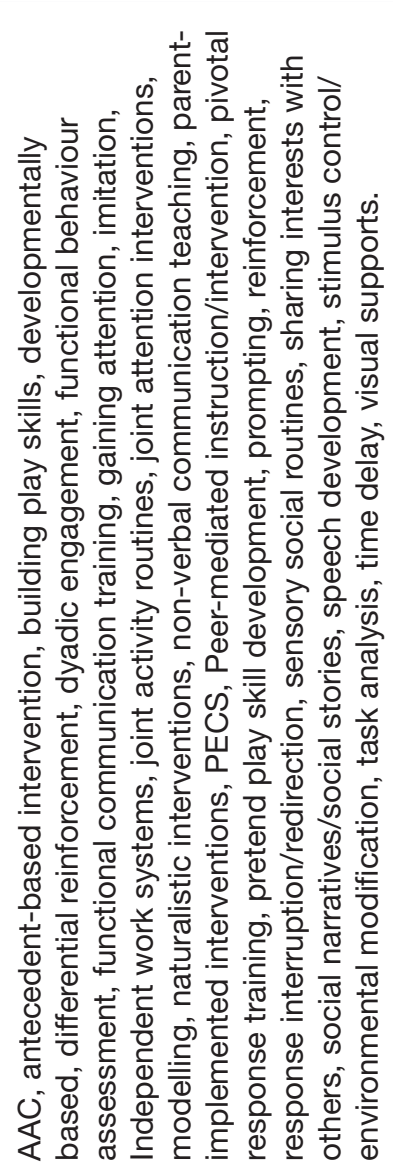

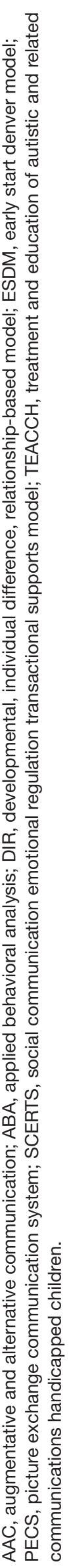

study to provide reliable screening for ASD and as a measure of change over time. ${ }^{18}$

Cognitive and language function

The Mullen Scales of Early Learning (MSEL ${ }^{19}$ ) assess development across key domains including language, motor and perceptual abilities. For each domain, raw scores and a corresponding age equivalence score is obtained.

\section{Adaptive function}

The Vineland Adaptive Behaviour Scales Second Edition ${ }^{20}$ (caregiver self-report form) is a standardised, normreferenced evaluation tool for children where parents will report on the child's functional level. One of the six ASELCCs used the structured interview form (equivalent scores are calculated for consistency with caregiver report form).

\section{Child behaviour}

The Child Behaviour Checklist for Ages 1.5-5 (CBCL $\left.1.5-5^{21}\right)$ and the Repetitive Behaviour Scale-Revised ${ }^{22}$ are completed by parents or caregivers regarding behavioural/emotional problems and restricted repetitive behaviours respectively. A Caregiver-Teacher Report Form version is also completed by the child's teacher or key worker at the ASELCC.

\section{Quality of life}

The Quality of Life in Autism ${ }^{23}$ questionnaire is also completed by the parents or caregivers. It is a 48 -item questionnaire assessing two subscales: Parents' overall perception of their quality of life; and, parents' perception of the degree to which their child's autism symptoms are problematic.

\section{Family and psychosocial factors}

The Parenting Stress Index-4 Short Form ${ }^{24}$ consists of 36 items measuring Parental Distress, Parent-Child Dysfunctional Interaction and Difficult Child domains. A family history questionnaire consisting of sociodemographic and family psychosocial factors as well as associated issues of relevance to the child and the family is also completed by the parent or caregiver.

\section{Data management}

A unique identifying number for each of the participating children is assigned by each ASELCC and used to establish a link between the various data collection forms, including parental questionnaires. All data will be managed using Research Electronic Data Capture (REDCap) tools hosted by the University of New South Wales, Sydney, Australia. ${ }^{25}$ REDCap is a secure, web-based software platform designed to support data capture for research studies.

\section{Data analysis plan}

Descriptive statistics will be used to show sociodemographic and clinical characteristics of participants at 
baseline, including frequencies, mean comparisons and measures of robustness. Bivariate and correlational analyses will be conducted to explore associations between sociodemographic variables and primary clinical outcomes. Multivariate analyses will be carried out to determine the impact of predictors on child developmental outcome measures such as the ADOS-2 calibrated severity score and subscales of the MSEL. Path analysis within a structural equation modelling (SEM) framework will be conducted to evaluate whether family, psychosocial and quality of life determinants mediate child outcomes. ${ }^{26}$ The SEM model will be designed specifically to test the following paths: (1) From clinical characteristics to child outcomes; (2) from family, psychosocial and quality of life variables to child outcomes and (3) indirect paths from factors listed in (4) via measures listed in (1) to child outcomes. Statistical analysis will be carried out by using SPSS V.26, MPLUS V.7.1 and the R language. ${ }^{27}$

\section{Ethics statement}

Ethical approval for the study was provided by human research ethics committees at the University of New South Wales (HC14267) as the lead site. This was also ratified by the respective academic institutions linked to the ASELCCs in each of the other five states.

\section{Dissemination of results}

Data will be stored at the ASELCC where the data is collected. Electronic records of data will be managed using REDCap. Results will be published in peer-reviewed journals and presented at conferences. A report summarising data and the interpretation of data will be published.

\section{DISCUSSION}

Improving the understanding of predictors of outcome following intervention is expected to elucidate the heterogeneity in ASD; in turn finding clinical characteristics that may form homogeneous subgroups that show similar baseline profiles, course, and intervention outcomes. While behavioural factors are related to outcomes, the fact that behaviourally similar children can respond very differently to the same intervention based on neurocognitive variables ${ }^{8}$ suggests that there are unique factors that determine the course, intervention response and longitudinal trajectory in ASD. One model relating these early atypicalities in brain development to the characteristic socio-communicative impairments has hypothesised that early low-level differences in recognition and orientation towards social stimuli leads to lack of social engagement with primary caregivers during infancy. This results in decreased engagement with caregivers and exposure to the reciprocal social interactions critical for healthy development of brain circuits that direct social behaviour. ${ }^{9}$ A key feature of early intervention is that it allows harnessing maximum benefit from the greater biological plasticity of the brain during the early years of life.
The findings from this study on the associated developmental, behavioural and adaptive profile that predict intervention outcomes will significantly improve our understanding of the determinants of a child's response to EII. This is expected to assist the identification and validation of accurate subtypes of autism which in turn will allow future research on underlying aetiologies associated with the subtypes, as well as inform research into longitudinal developmental trajectories in autism. The findings will be of national and international significance. Further, the evidence base on subtypes of autism will expedite decision making about early diagnosis and pave the way for a scientific approach to the understanding of how precision care can be applied in autism intervention, with consequent personal, social and economic benefits.

\section{Author affiliations}

${ }^{1}$ School of Psychiatry, Faculty of Medicine, University of New South Wales, Sydney, New South Wales, Australia

${ }^{2}$ School of Psychology and Public Health, La Trobe University, Olga Tennison Autism Research Centre, Director \& Chair, Melbourne, Victoria, Australia

${ }^{3}$ Director of Therapy and Clinical Services, Autism Association of Western Australia Inc, Subiaco, Western Australia, Australia

${ }^{4}$ Manager of Early Childhood Services, Autism Association of Western Australia Inc, Subiaco, Western Australia, Australia

${ }^{5}$ General Manager Operations, North West Tasmania Autism Specific Early Learning and Care Centre, St Giles Society Limited, Burnie, Tasmania, Australia

${ }^{6}$ Disability, Mental Health and Child Care Services, Anglicare SA, Daphne Street Autism Specific Early Learning and Care Centre, Adelaide, South Australia, Australia ${ }^{7}$ Chancellor's Postdoctoral Research Fellow, University of Technology Sydney Faculty of Health, Sydney, New South Wales, Australia

${ }^{8}$ Medical Director Child and Youth Community Health Services, Medical Director Child Development Program, SMO Sleep Medicine, Children's Health Queensland

Hospital and Health Service, South Brisbane, Queensland, Australia

${ }^{9}$ Child Development Unit, The Sydney Children's Hospitals Network Randwick and Westmead, Westmead, New South Wales, Australia

${ }^{10}$ Griffith Institute for Educational Research, Griffith University, Brisbane,

Queensland, Australia

${ }^{11}$ Autism Specific Early Learning and Care Centre - AEIOU for Children with Autism (AEIOU Foundation), Brisbane, Queensland, Australia

${ }^{12}$ Adjunct Senior Research Fellow, Griffith Institute for Educational Research Griffith University Nathan Campus, Nathan, Queensland, Australia

${ }^{13}$ Department of Paediatrics, Monash University, Clayton, Victoria, Australia ${ }^{14}$ Department of Paediatrics, Monash Children's Hospital, Clayton, Victoria, Australia

${ }^{15}$ South Western Sydney Local Health District, Academic Unit of Child Psychiatry, Liverpool Hospital, Sydney, New South Wales, Australia

${ }^{16}$ Ingham Institute for Applied Medical Research, Liverpool, New South Wales, Australia

Acknowledgements The authors acknowledge the Autism Specific Early Learning and Care Centres and the Service Providers and researchers who are contributing to the research program or have contributed over different time periods (in alphabetical order based on state location): South Western Sydney, NSW: KU Marcia Burgess Autism Specific Early Learning and Care Centre (KU Children's Services) and Ms Elizabeth Aylward; Brisbane, Queensland: Autism Specific Early Learning and Care Centre - AEIOU for Children with Autism (AEIOU Foundation) and Dr Jessica Paynter; Adelaide, SA: Anglicare - SA, Daphne Street Child Care and Specialist Early Learning Centre (Anglicare South Australia Inc); North West Tasmania: North West Tasmania Autism Specific Early Learning and Care Centre (St Giles Society) and Dr Colleen Check, Ms Miranda Stephens and Dr Damhnat McCann; Melbourne, Victoria: Margot Prior ASELCC, La Trobe University Community Childrens Centre (La Trobe University) and Dr Giacomo Vivanti and Dr Kristelle Hudry; Perth, WA: First Steps Autism Day Care (The Autism Association of Western Australia) and Dr Annette Jooston and Dr Nigel Chen.The authors acknowledge the financial support of the Cooperative Research Centre for Living with Autism (Autism CRC), established and supported under the Australian Government's 
Cooperative Research Centres Program. The authors acknowledge access to the Child and Family Outcome Study data that will be collected from children and family's attending the six ASELCCs established through funding from the Australian Governments Department of Social Services.

Contributors VE conceptualised the study. VE, CD, TA, KC, KF, GF, RG, MT and $\mathrm{KW}$ participated in the initial study design. VE and $C D$ obtained the research funding. VE, CD, TA, KC, KF, GF, RG, MT, KW, AM, HH, NS and RS contributed to subsequent amendments to the study protocol. VE and AM wrote the first draft of the manuscript. All authors (VE, CD, TA, KC, KF, GF, RG, MT, KW, AM, HH, NS and RS) contributed to subsequent revisions and approved the final manuscript.

Funding Financial support was received from the Cooperative Research Centre for Living with Autism (Autism CRC), established and supported under the Australian Government's Cooperative Research Centres Program.

Competing interests None declared.

Patient and public involvement Patients and/or the public were involved in the design, or conduct, or reporting, or dissemination plans of this research. Refer to the Methods section for further details.

Patient consent for publication Not required.

Provenance and peer review Not commissioned; externally peer reviewed.

Open access This is an open access article distributed in accordance with the Creative Commons Attribution Non Commercial (CC BY-NC 4.0) license, which permits others to distribute, remix, adapt, build upon this work non-commercially, and license their derivative works on different terms, provided the original work is properly cited, appropriate credit is given, any changes made indicated, and the use is non-commercial. See: http://creativecommons.org/licenses/by-nc/4.0/.

\section{ORCID iDs}

Anne Masi http://orcid.org/0000-0002-3135-6961

Valsamma Eapen http://orcid.org/0000-0001-6296-8306

\section{REFERENCES}

1 American Psychiatric Association. Diagnostic and statistical manual of mental disorders: DSM-5 $5^{\mathrm{TM}}$. 5th ed. Arlington, VA, US: American Psychiatric Publishing, Inc, 2013: 1-947.

2 Maenner MJ, Shaw KA, Baio J, et al. Prevalence of autism spectrum disorder among children aged 8 years - autism and developmental disabilities monitoring network, 11 sites, United States, 2016. MMWR Surveill. Summ. 2020;69:1-12.

3 Baird G, Simonoff E, Pickles A, et al. Prevalence of disorders of the autism spectrum in a population cohort of children in South Thames: the special needs and autism project (SNAP). The Lancet 2006;368:210-5.

4 Nicholas JS, Carpenter LA, King LB, et al. Autism spectrum disorders in preschool-aged children: prevalence and comparison to a schoolaged population. Ann Epidemiol 2009;19:808-14.

5 Masi A, DeMayo MM, Glozier N, et al. An overview of autism spectrum disorder, heterogeneity and treatment options. Neurosci Bull 2017;33:183-93.

6 Eapen V, Clarke RA. Autism spectrum disorders: from genotypes to phenotypes. Front Hum Neurosci 2014;8:914.
7 Doshi-Velez F, Ge Y, Kohane I. Comorbidity clusters in autism spectrum disorders: an electronic health record time-series analysis. Pediatrics 2014;133:e54-63.

8 Vivanti G. Predictors of treatment outcomes in toddlers with autism spectrum disorders. Neuropsychiatr Enfance Adolesc 2012;60:S128.

9 Dawson G. Early behavioral intervention, brain plasticity, and the prevention of autism spectrum disorder. Dev Psychopathol 2008;20:775-803.

10 Napolitano DA, Smith T, Zarcone JR, et al. Increasing response diversity in children with autism. J Appl Behav Anal 2010;43:265-71.

11 Eapen V, Crncec R, Walter A. There are gains, but can we tell for whom and why? predictors of treatment response following group early start denver model intervention in preschool - aged children with autism spectrum disorder. Autism 2016;06:1.

12 Fountain C, Winter AS, Bearman PS. Six developmental trajectories characterize children with autism. Pediatrics 2012;129:e1112-20.

13 Karst JS, Van Hecke AV. Parent and family impact of autism spectrum disorders: a review and proposed model for intervention evaluation. Clin Child Fam Psychol Rev 2012;15:247-77.

14 Council of Australian Governments. Investing in the early years - a national early childhood development strategy: Council of Australian Governments 2009.

15 Lord C, Rutter M, DiLavore PC. ADOS-2: autism diagnostic observation schedule. Los Angeles, CA: Western Psychological Services, 2012

16 Luyster R, Gotham K, Guthrie W, et al. The autism diagnostic observation Schedule-Toddler module: a new module of a standardized diagnostic measure for autism spectrum disorders. $J$ Autism Dev Disord 2009;39:1305-20.

17 Rutter M, Bailey A, Lord C. The social communication questionnaire: manual. Western Psychological Services, 2003.

18 Chandler S, Charman T, Baird G, et al. Validation of the social communication questionnaire in a population cohort of children with autism spectrum disorders. J Am Acad Child Adolesc Psychiatry 2007;46:1324-32.

19 Mullen E. Mullen scales of early learning. AGS edn. Circle Pines, MN: American Guidance Services Inc, 1995.

20 Sparrow S, Cicchetti D, Balla D. Vineland adaptive behavior scales:(Vineland II), survey interview form/caregiver rating form. Livonia, MN: Pearson Assessments, 2005.

21 Achenbach T, Rescorla L. Child behavior checklist for ages 1 1/2 - 5. System of empirically based assessment. Burlington, VT: University of Vermont, 2000.

22 Lam KSL, Aman MG. The repetitive behavior Scale-Revised: independent validation in individuals with autism spectrum disorders. J Autism Dev Disord 2007;37:855-66.

23 Eapen V, Crncec R, Walter A, et al. Conceptualisation and development of a quality of life measure for parents of children with autism spectrum disorder. Autism Res Treat 2014;2014:1-11. doi:10.1155/2014/160783

24 Abidin RR. Parenting stress index. 4th ed. Lutz, FL: PAR, 2012.

25 Harris PA, Taylor R, Minor BL, et al. The REDCap Consortium: building an international community of software platform partners. $J$ Biomed Inform 2019;95:103208.

26 Marcoulides GAS RE. Advanced structural equation modeling: issues and techniques. Mahwah, NJ: Lawrence Erlbaum Associates, 1996.

27 IBMCorp. IBM SPSS statistics for windows, version 26.0. Armonk, NY: IBM Corp Released, 2019. 